PROCEEDINGS OF THE

AMERICAN MATHEMATICAL SOCIETY

Volume 133, Number 5, Pages 1371-1377

S 0002-9939(04)07646-4

Article electronically published on October 28, 2004

\title{
SAMPLING SETS AND CLOSED RANGE COMPOSITION OPERATORS ON THE BLOCH SPACE
}

\author{
PRATIBHA GHATAGE, DECHAO ZHENG, AND NINA ZORBOSKA
}

(Communicated by Joseph A. Ball)

Dedicated to Chandler Davis for his 75th birthday

\begin{abstract}
We give a necessary and sufficient condition for a composition operator $C_{\phi}$ on the Bloch space to have closed range. We show that when $\phi$ is univalent, it is sufficient to consider the action of $C_{\phi}$ on the set of Möbius transforms. In this case the closed range property is equivalent to a specific sampling set satisfying the reverse Carleson condition.
\end{abstract}

\section{INTRODUCTION}

An analytic function $f$ on $D$ is said to belong to the Bloch space if $\sup \left\{\left(1-|z|^{2}\right)\left|f^{\prime}(z)\right|\right\}$ over $D$ is finite. Such functions form a complex Banach space $B$ under the norm $\|f\|_{B}=\sup \left\{\left(1-|z|^{2}\right)\left|f^{\prime}(z)\right|, z \in D\right\}+|f(0)|$. Functions belonging to the little Bloch space $B_{0}$ (consisting of the closure of polynomials in $B)$ are characterized by the property: $\lim _{|z| \rightarrow 1}\left(1-|z|^{2}\right) f^{\prime}(z)=0$.

Observe that $\sup \left\{\left(1-|z|^{2}\right)\left|f^{\prime}(z)\right|, z \in D\right\}$ is a pseudonorm, which coincides with the Bloch-norm on the closed subspace of functions that vanish at the origin. In general it coincides with the quotient norm on $B / \mathcal{C}$ where $\mathcal{C}$ denotes the closed subspace of constant functions.

The following concept is what all our criteria are based on.

We say that a subset $H$ of $D$ is called a sampling set for the Bloch space $B$ if $\exists k>0$ such that $\sup \left\{\left(1-|z|^{2}\right)\left|f^{\prime}(z)\right|, z \in D\right\} \leq k \sup \left\{\left(1-|z|^{2}\right)\left|f^{\prime}(z)\right|, z \in H\right\}$ holds $\forall f \in B$.

This is equivalent to $H$ being a sampling set for the $L^{\infty}$ version of the weighted Bergman space, denoted by $A^{-1}[8$, p. 22]. There are other definitions of sampling set for the Bloch space, but this one suits our purpose the best.

For each $z$ belonging to the unit disk $D$, let $\phi_{z}$ denote the Möbius transformation of $D$, given by

$$
\phi_{z}(w)=\frac{z-w}{1-\bar{z} w},
$$

for $w \in D$. The pseudohyperbolic distance (between $z$ and $w$ ) on $D$ is defined by

$$
\rho(z, w)=\left|\phi_{z}(w)\right| .
$$

$D(a, s)$ stands for the set $\{z \in D, \rho(z, a)<s\}$.

Received by the editors November 7, 2003 and, in revised form, December 30, 2003.

2000 Mathematics Subject Classification. Primary 47B33.

(C)2004 American Mathematical Society 
This metric is Möbius-invariant and has the following property:

$$
1-\rho(z, w)^{2}=\frac{\left(1-|z|^{2}\right)\left(1-|w|^{2}\right)}{|1-\bar{z} w|^{2}}=\left(1-|z|^{2}\right)\left|\phi_{z}^{\prime}(w)\right| .
$$

If $\phi$ is a holomorphic self-map of $D$, and $\tau_{\phi}(z)=\frac{\left(1-|z|^{2}\right) \phi^{\prime}(z)}{1-|\phi(z)|^{2}}$, then it is a simple consequence of the Schwarz-Pick lemma that $0 \leq\left|\tau_{\phi}(z)\right| \leq 1$ [1, p. 2]. Hence the composition operator $C_{\phi}$ defined by $C_{\phi}(f)=f \circ \phi$ is a bounded operator from $B$ into $B$. Moreover, if $\phi \in B_{0}$, then $C_{\phi}$ maps $B_{0}$ into $B_{0}$.

The function $\tau_{\phi}$ figures strongly in the study of compact composition operators on the Bloch space. In particular, if $C_{\phi}$ is compact on $B_{0}$, then $\tau_{\phi}(z) \rightarrow 0$ as $|z| \rightarrow 1$ and if it is compact on $B$, then $\tau_{\phi}(z) \rightarrow 0$ as $|\phi(z)| \rightarrow 1$ [6].

\section{A NECESSARY AND SUFFICIENT CONDITION}

The following fact is easy to check, but since it is pivotal to our investigation, we state it formally.

Theorem 0. If $\phi(0)=0$, the composition operator $C_{\phi}$ is bounded below on $B$ (equivalently, has closed range on $B$ ) if and only if it is bounded below on the subspace of functions that vanish at the origin. This is equivalent to the condition that $\|f \circ \phi\|_{B / \mathcal{C}} \geq k\|f\|_{B / \mathcal{C}}$.

Remark 1. If $\phi(0)=a$ and $\psi=\phi_{a} \circ \phi$, then $C_{\phi}$ is bounded below on $B$ if and only if $C_{\psi}$ is bounded below on $B$. Moreover, $\tau_{\psi}=\tau_{\phi}$.

So we assume from now on that $\phi(0)=0$ and that $C_{\phi}$ is acting on the subspace of functions that vanish at the origin. It is natural that the sets $\Omega_{\varepsilon}=\left\{z,\left|\tau_{\phi}(z)\right| \geq \varepsilon\right\}$ and $G_{\varepsilon}=\phi\left(\Omega_{\varepsilon}\right)$ play a pivotal role in our investigation.

Theorem 1. The composition operator $C_{\phi}$ is bounded below on $B$ if and only if $\exists \varepsilon>0$ such that if $\Omega_{\varepsilon}=\left\{z \in D,\left|\tau_{\phi}(z)\right| \geq \varepsilon\right\}$, then $G_{\varepsilon}$ is a sampling set for $B$.

Proof. Assume that $G_{\varepsilon}$ is a sampling set for $B$, for some $\varepsilon>0$. Then $\forall f \in B$ with $f(0)=0$,

$$
\begin{aligned}
\|f\|_{B} & \leq k \sup \left\{\left(1-|\phi(z)|^{2}\right) \mid f^{\prime}\left(\phi(z) \mid, z \in \Omega_{\varepsilon}\right\}\right. \\
& \leq k \sup \left\{\left|\tau_{\phi}(z)\right|^{-1}\left(1-|z|^{2}\right) \mid f^{\prime}\left(\phi(z) \| \phi^{\prime}(z) \mid, z \in \Omega_{\varepsilon}\right\}\right. \\
& \leq k \varepsilon^{-1} \sup \left\{\left(1-|z|^{2}\right)\left|(f \circ \phi)^{\prime}(z)\right|, z \in D\right\} \\
& \leq k \varepsilon^{-1}\|f \circ \phi\|_{B} .
\end{aligned}
$$

Conversely suppose that $C_{\phi}$ is bounded below on $B$. Then $\exists k>0$ such that whenever $f(0)=0$ and $\|f\|_{B}=1, \sup \left\{\left(1-|z|^{2}\right)|(f \circ \phi) \prime(z)| \geq k\right.$.

Suppose $\|f\|_{B}=1, f(0)=0$ and choose $z_{f}$ such that $\left(1-\left|z_{f}\right|^{2}\right)\left|(f \circ \phi)^{\prime}\left(z_{f}\right)\right| \geq$ $k / 2$, i.e. $\left|\tau_{\phi}\left(z_{f}\right)\right|\left[\left(1-\left|\phi\left(z_{f}\right)\right|^{2}\right) \mid f^{\prime}\left(\phi\left(z_{f}\right) \mid\right] \geq k / 2\right.$. But each of the two factors is no larger than 1 . Hence each is at least as large as $k / 2$. Thus if $\varepsilon=k / 2$, then $G_{\varepsilon}$ is a sampling set for $B$.

A subset $H$ of $D$ is said to satisfy the reverse Carleson condition if $\exists s>0$ and $c>0$ such that $|D(a, s) \cap H| \geq c|D(a, s)|$ for all $a \in D$, or equivalently if $\int_{H}|f(z)|^{2} d A(z) \geq c \int_{D}|f(z)|^{2} d A(z) \forall f$, which are analytic and square integrable on $D$.

This definition and the techniques we use are found in [5]. 
We show that if $G_{\varepsilon}$ satisfies the reverse Carleson condition, then $G_{\varepsilon}$ is a sampling set for the Bloch space. In order to do that we need to note an equivalent form for the Bloch-norm. We include a short proof for completeness.

Observation 1. For an analytic function $f$ on $D$ with $f(0)=0$,

$$
\|f\|_{B}^{2} \approx \sup \left\{\int_{D}\left|f^{\prime}(z)\right|^{2}\left(1-\left|\phi_{a}(z)\right|^{2}\right)^{2} d A(z), a \in D\right\}
$$

Proof. If $a \in D$, then,

$$
\begin{aligned}
\int_{D}\left|f^{\prime}(z)\right|^{2}\left(1-\left|\phi_{a}(z)\right|^{2}\right)^{2} d A(z) & =\int_{D}\left|f^{\prime}(z)\right|^{2}\left(1-|z|^{2}\right)^{2}\left|\phi_{a}^{\prime}(z)\right|^{2} d A(z) \\
& \leq\left(\|f\|_{B}^{2}\right) \int_{D}\left|\phi_{a}^{\prime}(z)\right|^{2} d A(z) \leq\|f\|_{B}^{2} .
\end{aligned}
$$

In order to prove the reverse inequality, first choose $s>0$ such that $\rho(z, w)<s$ implies $\left|\left(1-|z|^{2}\right) f^{\prime}(z)-\left(1-|w|^{2}\right) f^{\prime}(w)\right| \leq \frac{1}{4} \forall f \in B$ with $\|f\|_{B} \leq 1$. See [2] Proposition 2]. Now given $f \in B$ with $\|f\|_{B}=1$ and $f(0)=0$, we have $\sup \{(1-$ $\left.\left.|z|^{2}\right)\left|f^{\prime}(z)\right|, z \in D\right\} \geq 1$. Choose $z_{f}$ such that $\forall z \in D\left(z_{f}, s\right),\left(1-|z|^{2}\right)\left|f^{\prime}(z)\right| \geq \frac{1}{4}$.

Hence

$$
\begin{aligned}
& \int_{D}\left|f^{\prime}(z)\right|^{2}\left(1-\left|\phi_{z_{f}}(z)\right|^{2}\right)^{2} d A(z) \\
& \geq \int_{D\left(z_{f}, s\right)}\left|f^{\prime}(z)\right|^{2}\left(1-\left|\phi_{z_{f}}(z)\right|^{2}\right)^{2} d A(z) \\
& \geq \frac{1}{16} \int_{D\left(z_{f}, s\right)}\left(\frac{1-\mid \phi_{z_{f}}(z)^{2}}{1-|z|^{2}}\right)^{2} d A(z) .
\end{aligned}
$$

Note that $z \in D\left(z_{f}, s\right) \Rightarrow\left(1-|z|^{2}\right) \approx c_{s}\left(1-\left|z_{f}\right|^{2}\right)$ and $\left|\phi_{z_{f}}(z)\right|=\left|\rho\left(z_{f}, z\right)\right|<s$. Thus $\int_{D}\left|f^{\prime}(z)\right|^{2}\left(1-\left|\phi_{z_{f}}(z)\right|^{2}\right)^{2} d A(z) \geq c_{s}$.

Proposition 1. If $H \subseteq D$ and $H$ satisfies the reverse Carleson condition, then $H$ is a sampling set for the Bloch space.

Proof. Suppose $\left\|f_{n}\right\|_{B} \leq 1, f_{n}(0)=0$ and $\delta_{n}=\sup \left\{\left(1-|z|^{2}\right) \mid f_{n}^{\prime}(z), z \epsilon H\right\} \rightarrow$ 0 as $n \rightarrow \infty$.

If $a \in D$, then

$$
\begin{aligned}
\int_{D}\left|f_{n}^{\prime}(z)\right|^{2}\left(1-\left|\phi_{a}(z)\right|^{2}\right)^{2} d A(z) & \\
& =\int_{D}\left(1-|z|^{2}\right)^{2}\left|f_{n}^{\prime}(z)\right|^{2}\left|\phi_{a}^{\prime}(z)\right|^{2} d A(z) \\
& \leq c \int_{H}\left(1-|z|^{2}\right)^{2}\left|f_{n}^{\prime}(z)\right|^{2}\left|\phi_{a}^{\prime}(z)\right|^{2} d A(z)[6] \text { p. 10] } \\
& \leq c \delta_{n} \int_{H}\left|\phi_{a}^{\prime}(z)\right|^{2} d A(z) \leq c \delta_{n} \int_{D}\left|\phi_{a}^{\prime}(z)\right|^{2} d A(z) \leq c \delta_{n} .
\end{aligned}
$$

Hence, $\left\|f_{n}\right\|_{B}^{2} \leq c \delta_{n}$ (by the observation above), and $\left\|f_{n}\right\|_{B} \rightarrow 0$.

Our next proposition is an expanded form of the main result in [2]. We include a short proof of a part of it for completeness.

Proposition 2. Suppose $\phi$ is an analytic self-map of $D$ and assume that $\left\|\phi_{w} \circ \phi\right\|_{B / \mathcal{C}} \geq k \forall w \in D$. Then the following conditions hold. 
(1) Whenever $\varepsilon<k, \rho\left(z, G_{\varepsilon}\right) \leq \sqrt{1-\varepsilon}=r \forall z \in D$.

(2) Moreover, $\exists$ constants $s$ and $r^{\prime}, 0<s<1$ and $r^{\prime} \in[r, 1)$ such that given $w \in D \exists z_{w} \in D$ such that $\phi\left(D\left(z_{w}, s\right)\right) \subseteq D\left(w, r^{\prime}\right) \cap G_{\varepsilon}$.

Proof. (1) Suppose that $\varepsilon<k$ and $w \in D$. Choose $z_{w} \in D$ such that

$$
\left(1-\left|z_{w}\right|^{2}\right)\left|\phi_{w}^{\prime}\left(\phi\left(z_{w}\right)\right)\right|\left|\phi^{\prime}\left(z_{w}\right)\right| \geq \varepsilon \text {. }
$$

But $\left(1-\left|z_{w}\right|^{2}\right)\left|\phi_{w}^{\prime}\left(\phi\left(z_{w}\right)\right) \phi^{\prime}\left(z_{w}\right)\right|=\mid\left(\tau_{\phi}\left(z_{w}\right) \mid\left(1-\rho^{2}\left(w, \phi\left(z_{w}\right)\right)\right)\right.$. Each factor on the right-hand side is no larger than 1 ; hence each is at least $\varepsilon$. Thus $z_{w} \in \Omega_{\varepsilon}$ and $\rho\left(w, \phi\left(z_{w}\right)\right) \leq r<1$ where $r=\sqrt{1-\varepsilon}$.

(2) In [3, Theorem 6] it is shown that $\tau_{\phi}$ is Lipschitz with respect to the pseudohyperbolic metric on the domain and the Euclidean one on the range. We denote the Lipschitz constant by $\alpha$. Fix $\varepsilon^{\prime}<\varepsilon$, choose $s<\frac{\varepsilon-\varepsilon^{\prime}}{\alpha}$ and let $s<\varepsilon /(2 \alpha)$. If $\lambda \in D\left(z_{w}, s\right)$, then $\left|\tau_{\phi}(\lambda)\right| \geq \varepsilon^{\prime}$ and (by the Schwarz-Pick lemma) $\rho\left(\phi\left(z_{w}\right), \phi(\lambda)\right) \leq$ $\rho\left(z_{w}, \lambda\right)<s$. Thus for $\lambda$ in $D\left(z_{w}, s\right)$ we have

$$
\rho(w, \phi(\lambda)) \leq \frac{\rho\left(\phi\left(z_{w}\right), w\right)+\rho\left(\phi\left(z_{w}\right), \phi(\lambda)\right)}{1+\rho\left(w, \phi\left(z_{w}\right)\right) \rho\left(\phi\left(z_{w}\right), \phi(\lambda)\right)}<\frac{r+s}{1+r s}
$$

since $\frac{r+s}{1+r s}$ is an increasing function of $s$ and $r$ if they both lie in $(0,1)$. Let $r^{\prime}=\frac{r+s}{1+r s}$. We have shown that $\phi\left(D\left(z_{w}, s\right)\right) \subseteq D\left(w, r^{\prime}\right)$. By the choice of $s,\left|\tau_{\phi}(\lambda)\right| \geq \varepsilon^{\prime} \forall \lambda \in$ $D\left(z_{w}, s\right)$, i.e. $D\left(z_{w}, s\right) \subseteq \Omega_{\varepsilon^{\prime}}$ and hence $\phi\left(D\left(z_{w}, s\right)\right) \subseteq G_{\varepsilon^{\prime}}$.

We conclude that $\phi\left(D\left(z_{w}, s\right)\right) \subseteq G_{\varepsilon^{\prime}} \cap D\left(w, r^{\prime}\right)$.

In 2 it is shown that condition (1) of the previous proposition implies that $C_{\phi}$ is bounded below on the set of Möbius transforms, and that in case $C_{\phi}$ is close to being an isometry on the set of Möbius transforms $\left(k>\frac{15}{16}\right)$, then $C_{\phi}$ is bounded below on the Bloch space.

We now show that in case $\phi$ is univalent, then no lower bound on $k$ (except $k>0)$ is necessary.

\section{UNIVALENCE}

Corollary 1. If $\phi$ is a univalent self-map of $D$ and $\left\|\phi_{w} \circ \phi\right\|_{B / \mathcal{C}} \geq k \forall w \in D$, then $\forall \varepsilon<k, G_{\varepsilon}$ satisfies the reverse Carleson condition.

Proof. Let $\varepsilon<k$. Pick $\varepsilon^{\prime} \in(\varepsilon, k)$ and apply the conclusion of Proposition 2 to $\varepsilon^{\prime}$. $\exists s, r^{\prime} \in(0,1)$ such that given $w \in D \exists z_{w}$ such that $\phi\left(D\left(z_{w}, s\right)\right) \subseteq D\left(w, r^{\prime}\right) \cap G_{\varepsilon}$.

We use the fact that $\forall \lambda \in D\left(z_{w}, s\right),\left|\tau_{\phi}(\lambda)\right| \geq \varepsilon$ and the univalence of $\phi$ to conclude that $\left|\phi\left(D\left(z_{w}, s\right)\right)\right|=\int_{D\left(z_{w}, s\right)}\left|\phi^{\prime}(\lambda)\right|^{2} A(\lambda) \geq \varepsilon^{2} \int_{D\left(z_{w}, s\right)}\left(\frac{1-|\phi(\lambda)|^{2}}{1-|\lambda|^{2}}\right)^{2} d A(\lambda)$.

But $1-|\lambda|^{2} \approx c_{s}\left(1-\left|z_{w}\right|^{2}\right)$ and $1-|\phi(\lambda)|^{2} \approx c_{s}\left(1-\left|\phi\left(z_{w}\right)\right|^{2}\right) \approx c_{r} c_{s}\left(1-|w|^{2}\right)$ Since $|D(a, r)| \approx c_{r}\left(1-|a|^{2}\right)$, we have $\left|D\left(w, r^{\prime}\right) \cap G_{\varepsilon}\right| \geq\left|\phi\left(D\left(z_{w}, s\right)\right)\right| \geq c\left|D\left(w, r^{\prime}\right)\right|$ where $c$ is independent of $w$.

We summarize the main result for the univalent case in the following proposition.

Theorem 2. Suppose $\phi$ is a univalent self-map of D. Then the following are equivalent.

(1) $C_{\phi}$ is bounded below on $B$.

(2) $\left\|\phi_{w} \circ \phi\right\|_{B / \mathcal{C}} \geq k \forall w \in D$.

(3) $\forall \varepsilon<k, \rho\left(G_{\varepsilon}, z\right) \leq r<1 \forall z \in D, r$ depending only on $\varepsilon$.

(4) $\forall \varepsilon<k, G_{\epsilon}$ satisfies the reverse Carleson condition. 
Proof. $\quad(1) \Rightarrow(2)$ is obvious.

$(2) \Rightarrow(3)$ by Proposition 2 .

$(3) \Rightarrow(4)$ by Corollary 1.

(4) $\Rightarrow(1)$ by Proposition 1 .

Corollary 2. (1) If $\phi$ is univalent and $C_{\phi}$ is bounded below on BMOA, then it is bounded below on the Bloch space.

(2) If $\phi$ is univalent and $C_{\phi}$ is bounded below on the Bloch space, then it is bounded below on the Dirichlet space.

Proof. (1) If $\phi$ is univalent, then $\forall w \in D, \phi_{w} \circ \phi$ is univalent and in this case

$$
\left\|\phi_{w} \circ \phi\right\|_{B M O A} \approx\left\|\phi_{w} \circ \phi\right\|_{B}
$$

[10]. Hence if $C_{\phi}$ is bounded below on BMOA, then $\left\|\phi_{w} \circ \phi\right\|_{B} \geq k \forall w \in D$. Hence by Proposition $3, C_{\phi}$ is bounded below on the Bloch space.

(2) By Theorem 2, if $C_{\phi}$ is bounded below on the Bloch space, then $\exists \varepsilon>0$ such that $G_{\epsilon}$ satisfies the reverse Carleson condition; hence so does $G=\phi(D)$. By [4] $C_{\phi}$ is bounded below on the Dirichlet space.

Remark. W. Smith has given an example of a univalent map $\phi$ with $\overline{\phi(D)}=\bar{D}$ such that $\tau_{\phi}(z) \rightarrow 0$ as $|z| \rightarrow 1$ [9, 6.5]. Hence $C_{\phi}$ is compact on $B_{0}[\underline{6}]$. But $C_{\phi}$ has closed range on the Dirichlet space [4].

We now show that in case $\phi$ is univalent and $C_{\phi}$ is bounded below, then $G$ has no generalized cusps [7, p. 256] and in fact a somewhat stronger condition holds. We also give an example to show that it is not sufficient.

Observation 2. It is a simple consequence of Koebe's one-quarter theorem [7, p. 9] that if $\phi$ is univalent, then $\tau_{\phi} \approx \frac{\operatorname{dist}(\phi(z), \partial G)}{\operatorname{dist}(\phi(z), \partial D)}$.

Proposition 3. Suppose $\phi$ is univalent and there exists $\varepsilon>0$ such that $G_{\varepsilon}$ satisfies the reverse Carleson condition. Then there exists $\delta>0$ such that $\forall \omega \in \partial D$,

$$
\varlimsup_{\phi(z) \rightarrow w} \frac{\operatorname{dist}(\phi(z), \partial G)}{|\phi(z)-\omega|} \geq \delta .
$$

Proof. If $\eta<1$ let $\Delta(a, \eta)=\{z \in D,|z-a| \leq \eta(1-|a|)\}$ for $a \in D$. By [6. p. 4], $\exists \eta<1$ such that the following holds:

$$
G_{\varepsilon} \cap \Delta(a, \eta) \neq \emptyset \forall a \in D .
$$

Suppose $\omega \in \partial D$ and choose $\left\{a_{n}\right\}$ along the radius ending in $\omega$ such that $a_{n} \rightarrow \omega$. Choose $z_{n} \in \Omega_{\varepsilon}$ such that $\phi\left(z_{n}\right) \in \Delta\left(a_{n}, \eta\right)$. Then $\left|\phi\left(z_{n}\right)\right| \leq\left|a_{n}\right|+\eta\left(1-\left|a_{n}\right|\right) \leq$ $\eta+\left|a_{n}\right|(1-n)$ and hence $1-\left|\phi\left(z_{n}\right)\right| \geq(1-\eta)\left(1-\mid a_{n}\right) \mid$. Note that $\left|\omega-a_{n}\right|=1-\left|a_{n}\right|$. On the other hand, $\left|\phi\left(z_{n}\right)-\omega\right| \leq\left|\phi\left(z_{n}\right)-a_{n}\right|+\left|a_{n}-\omega\right| \leq \eta\left(1-\left|a_{n}\right|\right)+1-\left|a_{n}\right|=$ $(1+\eta)\left(1-\left|a_{n}\right|\right)$.

Now by observation 2 ,

$$
\begin{aligned}
\frac{\operatorname{dist}\left(\phi\left(z_{n}\right), \partial G\right)}{\left|\phi\left(z_{n}\right)-\omega\right|} & \geq \frac{1}{4} \frac{\tau_{\phi}\left(z_{n}\right)\left(1-\left|\phi\left(z_{n}\right)\right|\right.}{\left|\phi\left(z_{n}\right)-\omega\right|} \\
& \geq \frac{\varepsilon}{4} \frac{1-\eta}{1+\eta} .
\end{aligned}
$$


Next we give an example to show that the above condition is not sufficient for $C_{\phi}$ to be bounded below.

Example 1. Let

$$
G=D \backslash\left\{\bigcup_{i} D_{i} \cup l_{i}\right\}
$$

where $D_{i}=D\left(a_{i}, r_{i}\right)$ is the pseudohyperbolic disk with $\left|a_{i}\right|$ close enough to 1 , $r_{i} \rightarrow 1$ and $l_{i}$ is a line segment connecting $D_{i}$ to $\partial D$.

Let $\phi$ be the Riemann map onto $G$. If $\phi(z)$ approaches a point $\omega$ on $\partial D$ that is not an endpoint of the line segment $l_{i}$ or if $\omega \neq 1$, then $\tau_{\phi}(z) \approx 1$ by observation 2 . It is clear that the conclusion of the previous lemma holds for all $\omega \in \partial D$ that are not endpoints of line segments $l_{i}$ or 1 . For $\omega=1$, choose $\phi\left(z_{n}\right) \in G_{\varepsilon}$, approaching 1 non-tangentially, from below the $x$-axis.

For $\omega_{i}=$ endpoint of the line segment $l_{i}, G_{\varepsilon}$ has an extra non-tangential region taken away from $G$ but with the same angle opening for every $i$. Thus we may pick $\phi\left(z_{n}\right)$ approaching $\omega_{i}$ through an angle with a slightly larger opening. So the conclusion of the previous proposition is satisfied, but no pseudohyperbolic neighbourhood of $G_{\varepsilon}$ covers $D$. Hence $C_{\phi}$ is not bounded below on $B$.

The next example deals with a non-automorphic univalent $\phi$ that induces a closed-range composition operator on the Bloch space.

Example 2. Let $G=D \backslash[0,1)$, and let $\phi$ be the Riemann map onto $G$. Since

$$
\tau_{\phi}(z) \approx \frac{\operatorname{dist}(\phi(z), \partial G)}{1-|\phi(z)|}
$$

and the ratio on the right approaches 1 as $\phi(z)$ approaches any point $\omega \neq 1$ on the unit circle, $G_{\varepsilon}$ includes all of $D$, except a pseudohyperbolic neighbourhood of $[0,1)$. Hence with a suitable value of $r$, every point of $D$ is within pseudohyperbolic distance $r$ of $G_{\varepsilon}$ and hence $C_{\phi}$ is bounded below.

We are grateful to W. Smith for suggesting the example above and for helping us work through the details. We thank him for his kind interest and his patience with us. We are also grateful to J. Shapiro for helpful discussions and to the referee for the suggested improvements.

\section{REFERENCES}

1. J. Garnett, Bounded Analytic Functions, Academic Press, 1981. MR0628971|(83g:30037)

2. P. Ghatage, J. Yan and D. Zheng, Composition operators with closed range on the Bloch space, Proc. Amer. Math. Soc. 129 (2001), 2039-2044. MR1825915 (2002a:47034)

3. P. Ghatage and D. Zheng, Hyperbolic derivatives and generalized Schwarz-Pick Estimates, To appear in Proc. Amer. Math Soc..

4. M. Jovovic and B. MacCluer, Composition operators on Dirichlet spaces, Acta. Sci. Math. (Szeged) 63 (1997), 229-297. MR1459789 (98d:47067)

5. D. Luecking, Inequalities on Bergman Spaces, Ill. Jour. of Math. 25 (1981), 1-11. MR0602889 (82e:30072)

6. K. Madigan and A. Matheson, Compact composition operators on the Bloch space, Trans. Amer. Math. Soc. 347 (1995), 2679-2687. MR1273508 (95i:47061)

7. C. Pommerenke, Boundary Behaviour of Conformal Maps, vol. 299, Springer-Verlag, 1992. MR1217706 (95b:30008)

8. K. Seip, Beurling type density theorems in the unit disk, Invent. Math. 113, 21-29. MR 1223222 (94g:30033) 
9. W. Smith, Composition operators between Bergman and Hardy spaces, Trans. Amer. Math. Soc. 348 (1996), 2331-2348. MR1357404 (96i:47056)

10. D. Stegenga and K. Stephenson, A geometric characterization of analytic functions with bounded mean oscillation, J. London Math. Soc. 224 (1981), 243-254. MR0631937 (82m:30036)

Department of Mathematics, Cleveland State University, Cleveland, Ohio 44115

E-mail address: pghatge@csuohio.edu

Department of Mathematics, Vanderbilt University, Nashville, Tennessee 32740

E-mail address: zheng@math.vanderbilt.edu

Department of Mathematics, University of Manitoba, Winnipeg, Manitoba, Canada R3T2N2

E-mail address: zorboska@cc.umanitoba.CA 